\section{Associação entre presença de oclusopatias e insatisfação com a aparência dos dentes e gengivas: estudo com adolescentes brasileiros}

\section{Association between malocclusion and dissatisfaction with dental and gingival appearance: study with Brazilian adolescents}

\section{Carolina Marques Borges \\ Marco Aurélio Peres \\ Karen Glazer Peres}

Grupo de Estudos de Odontologia em Saúde Coletiva - GEOSC. Programa de Pós-Graduação em Saúde Coletiva do Centro de Ciências da Saúde da Universidade Federal de Santa Catarina.

Correspondência: Karen Glazer Peres. Centro de Ciências da Saúde, Departamento de Saúde Pú blica, Universidade Federal de Santa Catarina - Campus Universitário Trindade - Florianópolis, SC - CEP 88040-900. E-mail: karengp@ccs.ufsc.br

\section{Resumo}

Apesar da alta prevalência de oclusopatias em adolescentes ser mundialmente relatada, poucos estudos investigaram a associação entre oclusopatias definidas por critérios clínicos e a auto-avaliação da aparência dentária e das gengivas em adolescentes. $\mathrm{O}$ objetivo deste estudo foi investigar a associação entre oclusopatias e auto-avaliação da aparência dentária e gengival em adolescentes brasileiros. Foram utilizados os dados de um estudo transversal realizado com adolescentes de 15 a 19 anos de idade ( $n=16.126)$ de 250 cidades localizadas nas cinco macrorregiões do Brasil. O desfecho foi a insatisfação com a aparência dentária e gengival, sendo a principal variável exploratória as oclusopatias, medidas através do Índice de Estética Dental - DAI. As demais variáveis exploratórias, potenciais fatores de confusão e mediação, foram renda familiar per capita, atraso escolar, condição de estudo, sexo, idade, cor da pele, agravos bucais (cárie não tratada, perda dentária devido à cárie, cálculo, fluorose e dor nos dentes e gengivas) e a utilização dos serviços odontológicos. Realizaram-se análises de regressão de Poisson simples e multivariável. A prevalência de insatisfação com a aparência dos dentes e gengivas foi de 11,4\% (IC 95\%: 10,4-12,5). Todos os graus de oclusopatias foram associados à insatisfação com a aparência dos dentes e gengivas. A análise multivariável ajustada mostrou que a auto-avaliação negativa da aparência em adolescentes com oclusopatias graves ou muito graves foi $40 \%$ e $80 \%$ maior quando comparados àqueles com oclusão normal, respectivamente. Os resultados contribuem para a inclusão do critério de auto-avaliação da aparência dental durante as decisões de tratamento ortodôntico, principalmente no âmbito do Sistema Único de Saúde.

Palavras-chave: Saúde Bucal. Auto-avaliação. Adolescentes. Índice de Estética Dental. SB Brasil 2003. Oclusopatias. 


\section{Abstract}

In spite of the high prevalence of malocclusion in adolescents reported worldwide, there are few studies that have investigated the association between normative malocclusion and self-rated dental and gingival appearance among adolescents. The aim of this study was to identify the association between normative malocclusion and dissatisfaction with dental and gingival appearance among Brazilian adolescents. A cross-sectional study was carried out with adolescents aged 15 to 19 years ( $n=$ $16,126)$ living in 250 towns of all five Brazilian regions. Dissatisfaction with dental and gingival appearance was the outcome. The main explanatory variable was malocclusion assessed by using the Dental Aesthetic Index - DAI. The other explanatory variables included were per capita family income, schooling delay, study conditions, sex, age, skin color, dental outcomes (untreated dental caries, missing teeth due dental caries, dental calculus, fluorosis, and dental pain) and use of dental services. Simple and multivariable Poisson regression analyses were performed. Dissatisfaction with dental appearance reached $11.4 \%$ (95\%CI: 10.4-12.5) of the entire sample. All levels of malocclusion were associated with dissatisfaction with dental appearance. Adjusted multivariable analysis showed that dissatisfaction with dental appearance among individuals affected by severe or very severe malocclusion was respectively $40 \%$ and $80 \%$ higher than among those with normal occlusion. Malocclusion was associated with dissatisfaction with dental and gingival appearance. The results contribute to include self-rated dental appearance criteria in orthodontic treatment decision, mainly within the National Health System - SUS.

Keywords: Oral Health. Self-evaluation. Adolescents. Dental Aesthetic Index. SB Brasil 2003. Malocclusion.

\section{Introdução}

As oclusopatias são consideradas problemas de crescimento e desenvolvimento dos ossos maxilares e mandibulares durante a infância e a adolescência e podem acarretar alterações funcionais, estéticas ${ }^{1}$ ou psicossociais $^{2}$. Estas alterações podem impactar negativamente na qualidade de vida das pessoas ${ }^{3}$ devido ao isolamento social, baixa auto-estima, dificuldade de inserção no mercado de trabalho, estigmatização, chacota, constrangimento e timidez ${ }^{4}$.

Dentre as alterações que as oclusopatias podem acarretar, os aspectos estéticos têm sido apontados como os principais responsáveis por afetar negativamente a qualidade de vida dos indivíduos ${ }^{5}$, muitas vezes causando mais impacto do que outros aspectos físicos como o sobrepeso e a obesidade ${ }^{6,7}$.

Grande parte dos índices comumente utilizados para o diagnóstico e classificação das oclusopatias baseia-se em critérios essencialmente clínicos e ou epidemiológicos como, por exemplo, a antiga classificação proposta por Angle ${ }^{8}$ (1899) ou o Índice de Estética Dental (DAI) ${ }^{9}$. Entretanto, os instrumentos baseados exclusivamente em critérios normativos não consideram a opinião dos indivíduos sobre a própria saúde e/ou sobre a estética, e nem quais desvios de oclusão são percebidos como um problema para os mesmos ${ }^{5}$. A possibilidade de considerar a avaliação subjetiva do impacto das oclusopatias para a vida dos indivíduos em inquéritos epidemiológicos pode auxiliar na alocação de recursos públicos e na definição de grupos prioritários para tratamento ortodôntico no âmbito dos sistemas de saúde ${ }^{10}$. No Brasil, este enfoque torna-se ainda mais relevante ao se considerar a Política Nacional de Saúde Bucal, o Brasil Sorridente que, em caráter pioneiro no país, oferece à população tratamento odontológico especializado na rede pública, nos mais de 700 Centros de Especialidades Odontológicas, os CEOs ${ }^{11}$. Como complemento à atenção básica em saúde bucal, dentre outras especialidades odontológicas, alguns municípios tão díspares como Piri- 
piri (PI) e Rio de Janeiro (RJ) têm oferecido tratamento ortodôntico à população nos CEOs, adotando diferentes critérios para definição de prioridades para o tratamento e com dificuldades para a padronização dos mesmos $^{12}$.

Dentre os pressupostos do Brasil Sorridente destaca-se o uso da Epidemiologia com o objetivo de reorientar o modelo de atenção à saúde bucal, como, por exemplo, a realização de pesquisas epidemiológicas de base nacional. Em 2003 concluiu-se o projeto SBBrasil 2003, que proporcionou um dos mais completos diagnósticos da saúde bucal dos brasileiros. Nesta pesquisa, dentre outros aspectos, foram coletadas informações a respeito da prevalência e gravidade das oclusopatias em adolescentes, e também informações sobre a satisfação com a aparência dos dentes e gengivas deste grupo populacional ${ }^{13}$.

Desconhece-se a existência de estudos de âmbito nacional sobre a população brasileira que tenham investigado a associação entre a satisfação dos dentes e gengivas e a presença de oclusopatias. Frente às questões apontadas, o objetivo do presente estudo foi testar a associação entre a satisfação com a aparência dos dentes e gengivas e as oclusopatias em adolescentes brasileiros de 15 a 19 anos de idade, após o ajuste por potenciais variáveis de confusão. Assumiuse como hipótese que a insatisfação com a aparência dos dentes e gengivas está associada às oclusopatias, com um gradiente que segue a gravidade deste agravo.

\section{Métodos}

Este é um estudo transversal analítico que utilizou dados secundários referentes aos adolescentes brasileiros de 15 a 19 anos de idade $(n=16.126)$, residentes em 250 municípios brasileiros de todas as macrorregiões do país, oriundos do levantamento epidemiológico SB Brasil 2002-2003 ${ }^{13}$.

O Ministério da Saúde, juntamente com entidades odontológicas, Secretarias Estaduais e Municipais de Saúde, realizou nos anos de 2002 e 2003 o inquérito epide- miológico nacional SB Brasil, que investigou os principais agravos à saúde bucal dos brasileiros de diferentes grupos etários. $\mathrm{O}$ objetivo do SB Brasil foi realizar um amplo diagnóstico de saúde bucal de um país de dimensões continentais ${ }^{13}$.

Para a faixa etária dos 15 aos 19 anos, os dados foram coletados em domicílio e os adolescentes foram examinados quanto à presença e gravidade de oclusopatias, cárie dentária, condições periodontais, uso e necessidade de próteses e fluorose. Além dos exames odontológicos aplicou-se um questionário socioeconômico, sobre o uso de serviços odontológicos, presença de dor nos dentes e gengivas nos últimos 6 meses anteriores à entrevista, e autopercepção da saúde bucal, como a auto-avaliação da aparência dos dentes e gengivas, entre outras.

Para a seleção da amostra, foram realizadas duas pré-estratificações referentes às cinco macrorregiões brasileiras (Sul, Sudeste, Centro-Oeste, Norte e Nordeste) e ao porte populacional dos municípios ( $\leq$ 5.000 habitantes; de 5.001-10.000; de 10.001-50.000; 50.001-100.000 e > 100.000 habitantes). A amostragem foi probabilística por conglomerados realizada em três estágios: sorteio dos municípios, sorteio dos setores censitários/quadras/vilas e sorteio das residências. O tamanho da amostra do SB Brasil 2002-3 calculada para o grupo etário de 15 a 19 anos de idade foi igual a 19.910 indivíduos, obtendo-se uma taxa de resposta igual a $84,5 \%(n=16.833)$. Maiores informações sobre o inquérito nacional de saúde bucal SB Brasil 2002-2003 podem ser obtidas no seu relatório final ${ }^{13}$.

\section{Definição das variáveis}

\section{Desfecho}

A variável dependente investigada foi a auto-avaliação da aparência dos dentes e gengivas mensurada através da pergunta objetiva "Como você classifica a aparência de seus dentes e gengivas?", contida no questionário de autopercepção da saúde bucal utilizado pelo SB-Brasil 2002-3. As opções de respostas seguiram a escala de Likert (péssi- 
ma, ruim, regular, boa e ótima). Para análise estatística, a auto-avaliação da aparência dos dentes e gengivas foi dicotomizada em negativa, designada como insatisfação (ruim / péssima), e positiva, designada como satisfação (regular, boa, ótima).

\section{Exposição Principal}

A principal exposição estudada foi a presença de oclusopatias medida por meio do Índice de Estética Dental (Dental Aesthetic Index - DAI). O DAI leva em consideração dez diferentes critérios que recebem pesos específicos, a saber: perda de dentes incisivos, caninos ou pré-molares (peso 6), apinhamento ou espaçamento dentário no seguimento incisal (peso 1 cada), diastema (peso 3), irregularidade da maxila ou mandíbula no segmento anterior (peso 1 cada), overjet maxilar (peso 2) ou mandibular (peso 4), mordida aberta (peso 4) e relação anteroposterior de molar (peso 3). O resultado é obtido através da soma dos escores de cada característica encontrada e adicionada uma constante cujo valor é igual a 13. Esta somatória origina uma classificação que identifica a necessidade de tratamento ortodôntico dos indivíduos determinada pela gravidade das oclusopatias presentes, ou seja: oclusão normal ou com pequenos desvios (escores $\leq 25$ ), presença de oclusopatias definidas cujo tratamento é eletivo (escores entre 26 e 30), presença de oclusopatias graves com tratamento altamente desejável (escores entre 31 e 36) e presença de oclusopatias muito graves ou incapacitantes com prioridade máxima para o atendimento (escores $>36)^{14}$.

\section{Variáveis de confusão e mediação}

As demais variáveis independentes estudadas foram as condições socioeconômicas (renda per capita familiar, atraso escolar e condição de estudo), condições demográficas (sexo, idade e cor da pele), agravos à saúde bucal (cárie dentária não tratada, perda dentária devido à cárie, cálculo dentário, fluorose e dor nos dentes e gengivas) e a utilização dos serviços odontológicos (tempo decorrido desde a última consulta e tipo de serviço odontológico utilizado na última consulta).

A renda per capita familiar foi obtida pela soma de rendimentos de todos os moradores do domicílio, calculada em reais, dividindo-se pelo número total dos moradores e categorizada segundo sua distribuição em quartis ( $\leq 40$ reais, 41 a 80 reais, 81 a 150 reais e $>150$ reais).

A variável "atraso escolar" foi construída considerando-se o nível de educação formal (anos de escolaridade) dos adolescentes e a idade dos mesmos. Foram classificados como atrasados no estudo os adolescentes que, aos 18 e 19 anos de idade, possuíam até 10 anos de escolaridade; aqueles que aos 17 anos de idade apresentavam até nove anos de escolaridade, 16 anos de idade com até oito anos de escolaridade; adolescentes aos 15 anos de idade com até sete anos de escolaridade. Finalmente, o atraso escolar foi dicotomizado em não e sim.

A condição de estudo foi analisada considerando as seguintes categorias: estudante de escola privada, estudante de escola pública e adolescente que não estudava no período da entrevista. A opção "outros", verificada na variável "tipo de escola", foi considerada como sem informação ( $n=$ 125). A variável idade foi mantida como originalmente coletada no banco original (15, 16, 17, 18 e 19 anos de idade).

Para a cor da pele foi utilizada a autoclassificação, adotada pelo IBGE ${ }^{15}$ (branca, parda, preta, amarela e indígena). Para o presente estudo foram excluídos da análise os adolescentes amarelos e indígenas em razão de sua pequena proporção na amostra (3,8\% no conjunto).

A cárie dentária não tratada foi verificada através do componente $\mathrm{C}$ do índice CPOD (dentes permanentes cariados, perdidos e obturados) e categorizada segundo a sua distribuição em tercis $(0,1$ a 3 e $\geq 4)$. A perda dentária foi verificada através do componente $\mathrm{P}$ (dentes perdidos em virtude de cárie dentária) do índice CPOD e dicotomizada em zero e $\geq 1$ dente perdido. A presença de cálculo dentário foi verificada 
através dos escores do Índice Periodontal Comunitário (IPC), que avalia a condição periodontal segundo higidez, presença de sangramento gengival, cálculo dentário e bolsas periodontais. O cálculo dentário foi obtido a partir do índice CPI e, posteriormente, dicotomizado em não (ausente) e sim (presente). A categoria "não" considerou o CPI máximo igual 0 e a categoria "sim" o CPI máximo igual a 2. A variável fluorose foi construída a partir da variável original (normal, questionável, muito leve, leve e grave) e dicotomizada em ausente (normal) e presente (algum grau).

A dor nos dentes e gengivas foi dicotomizada em ausente (nenhuma dor) e presente (alguma dor).

No que se refere à utilização dos serviços odontológicos, o tempo decorrido desde a última consulta odontológica foi categorizado em nunca foi ao dentista, foi há menos de um ano, foi entre um e dois anos e há mais de dois anos. O tipo de serviço odontológico utilizado na última consulta foi dicotomizado em serviço privado (privado e planos de saúde privados) e público (Sistema Único de Saúde - SUS). Os serviços filantrópicos e outros tipos de serviços foram excluídos da análise porque não se pode afirmar se os mesmos eram conveniados ao SUS ou não $(2,4 \%)$.

\section{Análise dos dados}

Em um primeiro momento, o banco de dados original do SB-Brasil 2002-3, de domínio público e de livre acesso, foi capturado na página eletrônica do Ministério da Saúde do Brasil ${ }^{16}$ e exportado para o programa estatístico STATA 9.0, onde foram realizadas todas as análises. A estatística descritiva consistiu no cálculo das prevalências e intervalos de confiança (95\%) e o teste do qui-quadrado foi utilizado na análise bivariada entre desfecho e as variáveis independentes. As variáveis independentes foram agrupadas em socioeconômicas, demográficas, agravos bucais e utilização dos serviços odontológicos. As análises brutas e ajustadas foram realizadas por meio da regressão de Poisson - que apresenta como medida de associação a razão de prevalências. Esse tipo de análise é encorajado em casos de desfecho considerados comuns (>10,0\%), visando reduzir vieses na medida de associação como a superestimação ou subestimação quando a razão for maior ou menor que 1 , respectivamente ${ }^{17,18}$.

As variáveis que apresentaram valores de $\mathrm{p}<0,25$ no teste do qui-quadrado foram incluídas na análise múltipla seguindo a seqüência dos grupos mencionados. Durante o processo de modelagem foram mantidas as variáveis com valores de $\mathrm{p}<0,05$ ou aquelas que ajustavam o modelo. Todas as análises consideraram o efeito do delineamento amostral por meio do conjunto de comandos svy destinado à análise de dados oriundos de amostras complexas.

\section{Questões éticas}

O inquérito sobre as condições de saúde bucal da população brasileira - SB-Brasil 2002-3 - foi aprovado pelo Comitê Nacional de Ética em Pesquisas com Seres Humanos, sob o número 581/2000, em 21 de julho de 2000. O termo de consentimento livre e esclarecido foi obtido de todos os indivíduos participantes do estudo ${ }^{13}$.

\section{Resultados}

A amostra final analisada foi de 16.126 adolescentes, sendo $41,8 \%$ do sexo masculino. Do total de adolescentes investigados, 45,7\% auto-referiram serem pardos; $43,8 \%$ brancos e $10,5 \%$ pretos. Dentre a faixa etária estudada, a maior proporção $(28,5 \%)$ foi de adolescentes com 15 anos de idade (Tabela 1).

Cerca de um terço dos adolescentes apresentava quatro ou mais dentes com cáries não tratada e 35,6\% relataram episódio de dor nos dentes e/ou gengivas nos últimos seis meses. A maioria dos adolescentes (66,3\%) foi atendida pelo Sistema Único de Saúde (SUS) na última consulta odontológica. As oclusopatias graves e muito graves acometeram 14,0\% (IC 95\%: 13,1-14,9) e 
Tabela 1 - Distribuição da amostra, prevalência de insatisfação com a aparência dos dentes e gengivas, razão de prevalências brutas (RP) e intervalos de confiança (IC 95\%), segundo as variáveis socioeconômicas e demográficas. Brasil, 2002-2003.

Table 1 - Sample distribution, prevalence of appearance dissatisfaction, crude prevalence ratio (RP), and confidence intervals (95\% Cl), according to socioeconomic and demographic variables. Brazil, 2002-2003.

\begin{tabular}{|c|c|c|c|c|}
\hline Variável & $\begin{array}{c}\text { Amostra } \\
\mathrm{n}(\%)\end{array}$ & $\begin{array}{c}\text { Prevalência } \\
\text { Auto-avaliação negativa }\end{array}$ & $\begin{array}{l}\text { RP (IC95\%) } \\
\text { Bruta }\end{array}$ & $P$ \\
\hline Renda per capita em reais & & & & $<0,001^{1}$ \\
\hline$>150$ & $3.942(24,9)$ & $4,2(3,1-5,6)$ & 1,0 & \\
\hline $81-150$ & $3.767(23,8)$ & $6,0(5,1-7,1)$ & $1,4(1,2-1,7)$ & \\
\hline $41-80$ & $4.096(25,9)$ & $7,4(6,4-8,5)$ & $1,7(1,4-2,1)$ & \\
\hline$\leq 40$ & $4.005(25,4)$ & $9,3(7,7-11,2)$ & $2,1(1,7-2,7)$ & \\
\hline Atraso escolar & & & & $<0,001$ \\
\hline Não & $6.967(43,2)$ & $4,7(3,8-5,9)$ & 1,0 & \\
\hline Sim & $9.159(56,8)$ & $8,2(7,3-9,2)$ & $1,6(1,4-1,8)$ & \\
\hline Condição estudo & & & & $<0,001$ \\
\hline Escola privada & $742(4,7)$ & $2,5(1,6-3,8)$ & 1,0 & \\
\hline Escola pública & $10.945(69,1)$ & $5,8(5,2-6,6)$ & $2,2(1,5-3,3)$ & \\
\hline Não estuda & $4.147(26,2)$ & $10,1(8,412,1)$ & $3,4(2,3-4,9)$ & \\
\hline Sexo & & & & $<0,125$ \\
\hline Masculino & $6.736(41,8)$ & $6,3(5,4-7,3)$ & 1,0 & \\
\hline Feminino & $9.390(58,2)$ & $7,0(6,2-8,0)$ & $1,1(1,0-1,2)$ & \\
\hline Idade & & & & $<0,001$ \\
\hline 15 & $4.606(28,5)$ & $8,9(7,5-10,5)$ & 1,0 & \\
\hline 16 & $3.174(19,7)$ & $7,2(6,1-8,5)$ & $1,0(0,8-1,1)$ & \\
\hline 17 & $2.814(17,5)$ & $7,2(6,0-8,6)$ & $0,8(0,7-1,0)$ & \\
\hline 18 & $2.594(16,1)$ & $5,4(4,4-6,7)$ & $0,8(0,7-0,9)$ & \\
\hline 19 & $2.938(18,2)$ & $5,7(4,8-6,7)$ & $0,8(0,7-0,9)$ & \\
\hline Cor da pele & & & & $<0,001$ \\
\hline Branca & $7.071(43,8)$ & $5,1(4,2-6,2)$ & 1,0 & \\
\hline Parda & $7.369(45,7)$ & $8,0(7,1-8,9)$ & $1,5(1,3-1,7)$ & \\
\hline Preta & $1.686(10,5)$ & $8,1(6,3-10,4)$ & $1,5(1,2-1,8)$ & \\
\hline Total & $16.126(100,0)$ & $11,4(10,4-12,5)$ & & \\
\hline
\end{tabular}

$1 p$ de tendência linear.

1 linear trend $p$ value

18,8\% (IC 95\%: 17,6-20,0) dos indivíduos, respectivamente. (Tabela 2 ).

A prevalência da insatisfação com a aparência dos dentes e gengivas foi de 11,4\% (IC 95\%: 10,4-12,5).Verificou-se que, quanto menor a renda, maior a prevalência da autoavaliação negativa da aparência dos dentes e gengivas $(p<0,001)$. Adolescentes que apresentaram atraso escolar $(\mathrm{p}<0,001)$ e que não eram estudantes também apresen- taram maiores prevalências de insatisfação com a aparência $(\mathrm{p}<0,001)$, porém não foi observada diferença estatisticamente significativa entre os sexos (Tabela 1).

Quanto maior a gravidade das oclusopatias, maior a prevalência da insatisfação com a aparência $(p<0,001)$. Apresentar maior número de dentes com cárie não tratada, ter um ou mais dentes perdidos devido à cárie, apresentar cálculo dentário 
Tabela 2 - Distribuição da amostra, prevalência de insatisfação com a aparência dos dentes e gengivas, razão de prevalências brutas (RP) e intervalos de confiança (IC 95\%), segundo os agravos bucais e utilização dos serviços odontológicos. Brasil, 2002-2003.

Table 2 - Sample distribution, prevalence of appearance dissatisfaction, crude prevalence ratio (RP), and confidence intervals (95\%Cl), according to dental outcomes and utilization of dental services. Brazil, 2002-2003.

\begin{tabular}{|c|c|c|c|c|}
\hline Variável & $\begin{array}{c}\text { Amostra } \\
\mathrm{n}(\%)\end{array}$ & $\begin{array}{c}\text { Prevalência } \\
\text { Auto-avaliação negativa }\end{array}$ & $\begin{array}{l}\text { RP(IC95\%) } \\
\text { Bruta }\end{array}$ & $P$ \\
\hline Índice de Estética Dental & & & & $<0,001^{1}$ \\
\hline Oclusão normal & $7.525(46,7)$ & $5,9(4,9-7,0)$ & 1,0 & \\
\hline Oclusopatia definida & $3.313(20,5)$ & $6,6(5,6-7,8)$ & $1,4(1,2-1,6)$ & \\
\hline Oclusopatia grave & $2.260(14,0)$ & $6,3(5,3-7,5)$ & $1,5(1,2-1,8)$ & \\
\hline Oclusopatia muito grave & $3.028(18,8)$ & $9,3(8,1-10,7)$ & $2,1(1,8-2,5)$ & \\
\hline Cárie não tratada & & & & $<0,001^{1}$ \\
\hline 0 & $5.506(34,1)$ & $3,7(3,0-4,7)$ & 1,0 & \\
\hline $1-3$ & $5.638(34,9)$ & $5,3(4,4-6,5)$ & $1,7(1,4-2,0)$ & \\
\hline$\geq 4$ & $4.982(31,0)$ & $11,6(10,4-12,9)$ & $3,5(2,9-4,2)$ & \\
\hline Dentes perdidos & & & & $<0,001$ \\
\hline 0 & $10.241(63,5)$ & $5,8(5,0-6,8)$ & 1,0 & \\
\hline$\geq 1$ & $5.885(36,5)$ & $8,3(7,3-9,4)$ & $1,6(1,4-1,8)$ & \\
\hline Cálculo dentário² & & & & $<0,001$ \\
\hline Não & $7.441(57,9)$ & $4,7(3,9-5,8)$ & 1,0 & \\
\hline Sim & $5.405(42,1)$ & $9,0(8,0-10,2)$ & $2,3(2,0-2,7)$ & \\
\hline Fluorose dentária & & & & 0,850 \\
\hline Não & $14.164(89,9)$ & $11,4(10,3-12,6)$ & 1,0 & \\
\hline Sim & $1.578(10,1)$ & $11,1(9,5-13,1)$ & $1,0(0,8-1,2)$ & \\
\hline Dor dentes e/ou gengivas & & & & $<0,001$ \\
\hline Não & $10.282(64,4)$ & $3,0(2,6-3,5)$ & 1,0 & \\
\hline Sim & $5.687(35,6)$ & $13,3(11,7-15,1)$ & $2,5(2,3-2,8)$ & \\
\hline Tempo desde última consulta & & & & $<0,001^{1}$ \\
\hline$<1$ ano & $7.720(48,6)$ & $6,2(5,2-7,2)$ & 1,0 & \\
\hline $1-2$ anos & $3.710(23,3)$ & $5,4(4,5-6,6)$ & $1,1(1,0-1,3)$ & \\
\hline$\geq 3$ anos & $2.338(14,7)$ & $9,5(8,0-11,2)$ & $1,7(1,5-2,0)$ & \\
\hline Nunca foi & $2.122(13,4)$ & $8,3(6,8-10,1)$ & $1,7(1,4-2,0)$ & \\
\hline Tipo de serviço odontológico & & & & $<0,001$ \\
\hline Privado & $4.525(33,7)$ & $5,2(4,2-6,5)$ & 1,0 & \\
\hline Público (SUS) & $8.885(66,3)$ & $7,2(6,2-8,3)$ & $1,3(1,1-1,5)$ & \\
\hline
\end{tabular}

${ }^{1} \mathrm{P}$ de tendência linear; ${ }^{1}$ linear trend $p$ value.

${ }^{2}$ Excluiu-se dentes com sangramento gengival $(n=3.266)$. O maior valor perdido entre as variáveis foi para cálculo dental $(n=3.280) ;{ }^{2}$ teeth presenting gingival bleeding were excluded ( $n 3,266)$. The highest missing value was observed for dental calculus $(n=3,280)$

em pelo menos um sextante e ter sentido dor nos dentes e gengivas nos últimos seis meses foram associados positivamente à insatisfação com a aparência dos dentes e gengivas $(\mathrm{p}<0,01)$ (Tabela 2). Quanto maior o tempo decorrido desde a última consulta odontológica, maior a insatisfação com a aparência dos dentes e gengivas (Tabela 2).

$\mathrm{Na}$ análise ajustada, verificou-se que a prevalência de insatisfação com a aparência dos dentes e gengivas nos indivíduos com oclusopatias consideradas como defini- 
Tabela 3 - Associação entre Índice de Estética Dental e insatisfação com aparência de dentes e gengivas (razão de prevalências - RP). Análise de regressão múltivariável de Poisson. Brasil, 2002-2003.

Table 3 - Association between Dental Aesthetic Index and dissatisfaction with dental and gingival appearance (prevalence ratio-RP). Poisson multivariable regression analysis. Brazil, 2002-2003.

\begin{tabular}{|c|c|c|c|c|c|c|c|c|}
\hline Variável & $\begin{array}{c}\text { Modelo } 1 \\
\mathrm{RP}_{\mathrm{b}}(\mathrm{IC} 95 \%)\end{array}$ & $\mathrm{P}$ & $\begin{array}{c}\text { Modelo } 2 \\
\mathrm{RP}_{\mathrm{a}}(\mathrm{IC} 95 \%)\end{array}$ & $\mathrm{P}$ & $\begin{array}{c}\text { Modelo } 3 \\
\mathrm{RP}_{\mathrm{a}}(\mathrm{IC} 95 \%)\end{array}$ & $\mathrm{P}$ & $\begin{array}{c}\text { Modelo } 4 \\
\mathrm{RP}_{\mathrm{a}}(\mathrm{IC} 95 \%)\end{array}$ & $\mathrm{P}$ \\
\hline Índice de Estética Dental & & $<0,001$ & & $<0,001$ & & $<0,001$ & & $<0,001$ \\
\hline Oclusão normal & 1,0 & & 1,0 & & 1,0 & & 1,0 & \\
\hline Oclusopatia definida & $1,4(1,2-1,6)$ & & $1,3(1,2-1,6)$ & & $1,3(1,2-1,6)$ & & $1,3(1,1-1,5)$ & \\
\hline Oclusopatia grave & $1,5(1,2-1,8)$ & & $1,5(1,2-1,8)$ & & $1,5(1,2-1,8)$ & & $1,4(1,1-1,7)$ & \\
\hline Oclusopatia muito grave & $2,1(1,8-2,5)$ & & $2,0(1,8-2,4)$ & & $2,0(1,7-2,4)$ & & $1,8(1,5-2,2)$ & \\
\hline
\end{tabular}

$\mathrm{RP}_{\mathrm{b}}$ : Valor de RP bruto; $\mathrm{RP}_{\mathrm{a}}$ :Valor de $\mathrm{RP}$ ajustado.

Modelo 1: Análise bruta.

Modelo 2: Modelo 1 (Índice de Estética Dental) + renda familiar per capita + atraso escolar+ condição de estudo.

Modelo 3: Modelo $2+$ sexo + idade + cor da pele.

Modelo 4: Modelo 3 + cárie não tratada + cálculo dentário + dor de dente + tempo desde a última consulta + tipo de serviço utilizado.

$R P_{b}: R P$ crude value; $R P_{a}: R P$ adjusted value.

Model 1: Crude analysis.

Model 2: Model 1 (Dental Aesthetic Index) + per capita family income + schooling delay + study condition.

Model 3: Model $2+$ sex + age + skin color.

Model 4: Model 3 + untreated dental caries + dental calculus + dental pain + time since last dental visit + type of dental service used.

das, graves e muito graves foi $30 \%, 40 \%$ e $80 \%$ maior, respectivamente, do que nos indivíduos com oclusão normal (Tabela 3). Mantiveram-se associadas à auto-avaliação negativa da aparência dos dentes e gengivas apresentar renda per capita $\leq 40$ reais (RP: 1,5 IC 95\%: 1,1-2,0), maior número de dentes com cárie não tratada (RP: 2,1 IC 95\%: 1,7-2,6), presença de cálculo dentário em pelo menos um sextante (RP: 1,5 IC 95\%: $1,3-1,7)$, relato de dor nos dentes e gengivas (RP: 2,1 IC 95\%: 1,8-2,5) e nunca ter ido ao dentista (RP: 1,4 IC 95\%: 1,2-1,6) (dados não apresentados).

\section{Discussão}

O presente estudo investigou a associação entre a insatisfação com a aparência dos dentes e gengivas e as oclusopatias em adolescentes brasileiros. Desconhece-se estudo brasileiro de base populacional que tenha abordado esta temática.

A prevalência da insatisfação com a aparência dos dentes e gengivas nos adolescentes foi de $11,4 \%$ sendo menor quando comparada a países tão díspares como Tanzânia $(23,3 \%)^{19}$, Nigéria $(57,4 \%)^{2} \mathrm{e}$ Turquia $(42,7 \%)^{20}$. No entanto, as comparações devem ser feitas com cautela devido às diferenças metodológicas existentes, como as diferentes faixas etárias estudadas, tipos de índices utilizados ou formas de categorização das variáveis ${ }^{3}$. Além disso, o conceito de beleza e a percepção dos indivíduos quanto à estética facial varia conforme os padrões culturais vigentes em cada contexto $^{21}$. É importante ressaltar que o fato de a pergunta "Como classifica a aparência dos seus dentes e suas gengivas?" se referir a duas condições distintas pode ter dificultado a interpretação da mesma pelo respondente. Provavelmente, seria mais adequado obter informações sobre a auto-avaliação da aparência dentária e gengival separadamente.

Corroborando os resultados de outras pesquisas, os indivíduos mais pobres avaliaram pior suas condições dentárias e gengivais $^{22}$. Contrapondo outros estudos ${ }^{5,7}$, não houve diferenças na insatisfação com a aparência dentária e gengival quanto ao sexo.

A insatisfação com a aparência de dentes e gengivas foi associada às oclusopatias, mesmo quando consideradas outras morbidades como a cárie dentária. Problemas oclusais influenciando negativamente a qualidade de vida de adolescentes, independente da presença de outros agravos bucais, foram relatados em outro estudo no sul do Brasil ${ }^{23}$. 
Um dos principais achados deste estudo foi o aumento da proporção de indivíduos insatisfeitos com a aparência dos dentes e gengivas conforme o aumento da gravidade das oclusopatias. Este achado reforça a relevância de considerar as medidas subjetivas quando se pretende identificar os problemas oclusais que provocam impacto negativo na vida dos adolescentes ${ }^{19}$.

Considerando que o tratamento ortodôntico foi incluído no elenco de alternativas terapêuticas especializadas do Sistema Único de Saúde, o diagnóstico das oclusopatias possui dois objetivos principais: avaliar a necessidade e a prioridade de tratamento dos indivíduos, e obter informações para alocar adequadamente os recursos necessários para a provisão do tratamento ortodôntico para a população ${ }^{24}$. Segundo os resultados do presente estudo, o Índice de Estética Dental (DAI), quando utilizado conjuntamente com um critério subjetivo, pode ser considerado uma boa ferramenta epidemiológica para ser aplicada em inquéritos populacionais, assim como auxiliar na definição da priorização do atendimento ortodôntico, a fim de contribuir para a alocação de recursos públicos para a saúde ${ }^{25}$. O acesso a tratamento ortodôntico tem sido relatado na literatura científica como um dos fatores determinantes na autoavaliação positiva da aparência dentária ${ }^{20,26}$, reforçando a importância de se incorporar de forma efetiva e eficiente este tipo de tratamento no SUS.

As principais considerações metodológicas desta pesquisa dizem respeito ao índice DAI e ao delineamento amostral. Apesar do DAI ser um índice recomendado pela Organização Mundial da Saúde ${ }^{14}$ para investigar a necessidade de tratamento ortodôntico e a gravidade das oclusopatias, este índice inclui também a perda dentária de incisivos, caninos e pré-molares, condições que têm um importante peso na construção do mesmo. Entretanto, a proporção de indivíduos que apresentou alguma perda dos referidos elementos dentários foi pequena $(1,7 \%)$ e não interferiu expressivamente nos resultados finais.

A técnica de amostragem por conglo- merados e a forma de seleção da amostra garantiram a validade interna do estudo. Apesar de não ter sido realizado o cálculo dos pesos amostrais ${ }^{27}$, estima-se que isto não apresenta impacto ponderável nem na magnitude e, tampouco, na direção das medidas de associação estimadas nesta pesquisa $^{28}$. O desconhecimento da hipótese e dos objetivos do presente estudo pelos examinadores do SB-Brasil reduziu a possibilidade de viés de aferição.

As proporções de meninos e meninas na amostra (42,0\% e 58,0\%, respectivamente) foram diferentes daquelas observadas para a mesma faixa etária na população brasileira: $53,0 \%$ e $47,0 \%$, respectivamente ${ }^{29}$. Entretanto, outras variáveis como a renda, a proporção de adolescentes com atraso escolar e a condição de estudo distribuíram-se de maneira semelhante ao perfil da população brasileira ${ }^{29}$.

Outras limitações inerentes ao presente estudo se referem ao delineamento transversal e à utilização de dados secundários. Estudos transversais não permitem analisar a relação de causalidade entre as variáveis investigadas e o desfecho. Além disso, as limitações aumentam quando são utilizados dados secundários, pois a qualidade da coleta e a tabulação dos dados não são passíveis de serem controladas.

Apesar das limitações discutidas, há de se ressaltar os pontos fortes do presente estudo. A utilização de dados oriundos do último e maior inquérito de saúde bucal realizado do Brasil é importante para a valorização do trabalho realizado pelo Ministério da Saúde em parceria com entidades odontológicas assim como para o aperfeiçoamento de levantamentos futuros.

Nossos resultados apontam para a necessidade da continuidade dos estudos sobre a incorporação dos critérios subjetivos aos normativos visando reduzir as muitas lacunas existentes neste campo. Entender como e quais oclusopatias causam impacto negativo na vida das pessoas pode contribuir para definir os critérios para atendimento nos centros de especialidades odontológicas - os CEOs - do SUS. 


\section{Referências}

1. Samorodnitzky-Naveh GR, Geiger SB, Levin L. Patients' satisfaction with dental esthetics. J Am Dent Assoc 2007; 138(6): 805-8.

2. Onyeaso CO, Sanu OO. Psychosocial implications of malocclusion among 12-18 year old secundary school children in Iban, Nigéria. Odontostomatologie Trop 2005; 28 (109): 39-48.

3. Zhang M, McGrath C, Hägg U. Orthodontic treatment need and oral health-related quality among children. Community Dent Health 2009; 26(1): 58-61.

4. Klages U, Aladàr B, Yvette G, Andrei Z. Dental esthetics, orthodontic treatment and oral-health attitudes in Young adults. Am J Orthod Dentofacial Orthop 2005; 128(4): 442-9.

5. Marques LS, Ramos-Jorge ML, Paiva SM, Pordeus IA. Malocclusion: esthetic impact and quality of life among Brazilian schoolchildren. Am J Orthod Dentofacial Orthop 2006; 129(3): 424-7.

6. Shaw WC, Lewis HG, Robertson NRE. Perception of malocclusion. Br Dent J 1975; 138: 211.

7. Peres KG, Barros AJD, Anselmo L, Peres MA, Barros FC. Does malocclusion influence the adolescent's satisfaction with appearance? A cross-sectional study nested in a Brazilian birth cohort. Community Dent Oral Epidemiol 2008; 36(2): 137-43.

8. Angle, E H. Classification of Malocclusion. Dental Cosmos 41; 1899: 248-264, 350-357. p. 262.

9. Cons NC, Jenny J, Kohout FJ. DAI: the dental aesthetic index. Iowa City: College of Dentistry, University of Iowa; 1986.

10. Locker D, Berka E, Jokovic A, Tompson B. Does selfweighting of items enhance the performance of an oral health-related quality of life questionnaire? Community Dent Oral Epidemiol 2007; 35(1): 35-43.

11. Pucca GA. A política nacional de saúde bucal como demanda social. Ciência \& Saúde Coletiva 2006; 11(1): 243-6.

12. Ministério da Saúde. Departamento de Atenção Básica em saúde Bucal. I Encontro dos Centros de Especialidades Odontológicas. Disponível em: http:// dab.saude.gov.br/evento/ 1EncontroCeoEsf/. [Acessado em 27 de junho de 2009].

13. Brasil. Ministério da Saúde. Secretaria de Atenção à Saúde. Departamento de Atenção Básica. Coordenação de Saúde Bucal. Projeto SB Brasil 2003. Condições de saúde bucal da população brasileira: 2002-2003. Resultados principais. Brasília: Ministério da Saúde; 2004.

14. World Health Organization. Oral Health Surveys: basic methods. 4.ed. Geneva: WHO; 1997.
15. Instituto Brasileiro de Geografia e Estatística (IGBE). Pesquisa nacional por amostra de domicílios: acesso e utilização de serviços de saúde. Rio de Janeiro; 2000.

16. Ministério da Saúde. Cadernos de Atenção Básica. Disponível em: http://dtr2004.saude.gov.br/dab/ saudebucal/vigilancia.php. [Acessado em 27 de junho de 2009].

17. Barros AJ, Hirakata VN. Alternatives for logistic regression in cross-sectional studies: an empirical comparison of models that directly estimate the prevalence ratio. BMC Med Res Methodol 2003; 3: 21.

18. Vigo A. Modelando desfechos comuns: viés e precisão. Cad Saúde Pública 2006; 21(11): 2496-7.

19. Mtaya M, Astrom AN, Brudvik P. Malocclusion, psychosocial impacts and treatment need: A cross-sectional study of Tanzanian primary school-children. BMC Oral Health 2008; 8: 14.

20. Akarslan ZZ, Sadik B, Erten H, Karabulut E. Dental esthetic satisfaction, received and desired dental treatments for improvement of esthetics. Indian J Dent Res 2009; 20(2): 195-200.

21. Kiekensa RMA, Malthab JC, van 't Hofc MA, KuijpersJagtmand AM. Objective measures as indicators for facial esthetics in white adolescents. Angle Orthod 2006; 76(4): 551-56. Angle 1899.

22. Mandall NA, McCord JF, Blinkhorn AS, Worthington HV, $\mathrm{O}$ Brien KD. Perceived aesthetic impact of malocclusion and oral self-perceptions in 14-15-year-old Asian and Caucasian children in greater Manchester. Eur J Orthod 1999; 22(2): 175-83.

23. Peres KG, Peres MA, Araujo CLP, Menezes AMB, Hallal PC. Health and Quality of Life Outcomes 2009; 7: 95.

24. Oliveira CM. Maloclusão no contexto da saúde pública. In: Bonecker M, Sheiham A (org.). Promovendo saúde bucal na infância e na adolescência. São Paulo: Santos, 2004; 75-84.

25. Maciel SM, Kornis GEM. A ortodontia nas políticas públicas de saúde bucal: um exemplo de equidade na Universidade Federal de Juiz de Fora. Rev Saúde Coletiva 2006; 16(1): 58-81.

26. Svedström-Oristo AL, Pietilä T, Pietilä I, Vahlberg T, Alanen P, Varrela J. The Angle Orthodontist 2009; 79(3): 479-83.

27. Queiroz RC, Portela MC, Vasconcellos MT. Pesquisa sobre as Condições de Saúde Bucal da População Brasileira (SB Brasil 2003): seus dados não produzem estimativas populacionais, mas há possibilidade de correção. Cad Saúde Publica 2009; 25: 47-58. 
28. Narvai PC, Antunes JLF, Moysés SJ, Frazão P, Peres MA, Peres KG, Sousa MLR, Roncalli AG. Validade Científica de Conhecimento Epidemiológico Gerado com Base no Estudo Saúde Bucal Brasil 2003. Cad de Saúde Publica 2010; 26(4): 647-70.
29. Instituto Brasileiro de Geografia e Estatística (IGBE). Contagem da População 2007 [on line]. Rio de Janeiro, Brasil; 2004. Disponível em http://ibge.gov.br. [Acessado em 27 de junho de 2009]

Recebido em: 11/01/10

Versão final reapresentada em: 27/05/10

Aprovado em: 23/06/10 